più puro, più sereno che altrove e conseguentemente la rifrazione dovrà essere minore.

Avendo un discreto numero di misure di depressione posso prendendo la media di queste doterminare colla semplice formula

$$
n=\frac{2\left(d-d_{r}\right)}{d}
$$

il coeficiente di rifrazione marina $n$. Essendo $d=15,36$ $d_{r}$ medio $=14,53$ sarà

$$
n=0.1073
$$

valore poco discosto da quello ultimamente determinato nell'anno 1899 dal Prof. E. Soler a Palermo e dato da

$$
n=0,1026 \text {. }
$$

Dalla qual concordanza emerge che il metodo semplicissimo proposto dal Prof. Ricco per lo studio della variazione della rifrazione atmosferica si puó applicare per la determinazione del coefficiente di rifrazione marina in sostituzione di quelli complicati e difficoltosi adottati sino ad ora.

\title{
IIBRI NUOVI
}

\section{MANUALE DI BLETTROTECNIEA,}

\section{GRAWINKEL $e$ STREGKER.}

Edizigne italiana ampliata sulla sesta edizione tedesca, dall'ing FLAVIO DESSY.

(Ulrico Hoepli, Milano 1901)

E questo un manuale che merita di essere segnalato, come quello che racehiude, in uno spazio relativamente ristretto, i risultati di studi teorici e sperimentali di elettricità che si succedettero con rapidita meravigliosa, specialmente, in questi ultimi anni.

Esso non è un vero trattato di elettrotecnica, ma bensi un manuale pratico, interamente composto di nozioni generali, di 
numeri, di tabelle, di dati desunti, più che da altre pubblicazioni consimili, dall' esperienza personale degli A., e che si richiedono nelle operazioni ordinarie di un esercente.

Per la chiarezza dell' esposizione, per il modo coscienzioso con cui è stato compilato, sono ben sicuro, che questo manuale incontrerà largo favore fra gli studiosi di elettricità e sarà di guida preziosa a chi deve esercire o eseguire impianti elettrici.

Non nascondo che qua e là ci sono delle lacune, che, del resto, non possono mancare, data l' estensione della materia trattata.

Il libro è diviso in tre parti, di ciascuna delle quali credo opportuno dare un brevissimo cenno.

La prima parte è dedicata alle nozioni generali di magnetismo e di elettricità; vengono specialmente sviluppati e con molta chiarezza i principii e le leggi sull' elettromagnetismo e sulle correnti alternative. E quì, con molta opportunità, gli A. accennano alle equazioni di Lord Kelvin sulle correnti di scarica dei condensątori.

Nella seconda parte, dopo avere accennato al sistema assoluto di misure C. G. S. e allo grandezze magnetiche ed elettriche, svolgono ampliamente, e con ricco patrimonio di nozioni pratiche, la parte che riguarda i metodi e strumenti di misure. Un capitolo speciale è dedicato alle misurazioñ tecniche, ed un altro alle misurazioni fotometriche.

Nella terza parte gli A., richiamano le generalitá delle macchine dinamo elettriche, e danno norme, dati e consigli per la costruzione delle dinamo a corrente continua e alternativa.

Molto bene esposti, e ricchi di cognizioni pratiche sono i capitoli dedicati agli accumulatori, alla trasmissione e distribuzione della corrente, alla illuminazione elettrica, all' elettrochimica, alla telegrafia ed alla telefonia.

Infine gli A. danno un rapido cenno sugli orologi elettrici, sugli apparecchi registratori, sulla produzione elettrica del calore e sui parafulmini.

$\dot{E}$ aggiunta un'appendice contenente la legislazione italiana relativa agli impianti elettrici e le prescrizioni di sicurezza. 
L' edizione, come sempre, è nitida e ricca di bellissime incisioni. Soltanto dubito che il prezzo alquanto elevato del manuale, impedisca quella diffusione che merita.

Ing. G. Carro cao.

\section{EIVISTA}

Comptes Rendus. T. CXXXII, Gennaio a Aprile 1901.

LAGRANGE E. Sulla propagazione delle onde herziane nella telegrafia senza fili (pp. 203-204). - L'A. ha ricercato l' ufficio che la terra ha nella propagazione delle onde hertziane, all' infrori dell' influenza che essa ha sulla natura stessa delle onde prodotte. Avendo ricercato pereió se l'azione delle onde si faceva sentire su un coherer sotterrato nel suolo a $0 ", 30$ di profondità ne ha ottenuto un risultato completamente negativo. L'A. ne conclude che le onde non penetrano affat to nell' interno del suolo, e che $v i$ è probabilmente assorbimento $\theta$ riflessione delle onde emesse dall' antenna.

Rrcco A. e Janssers. Comunicazioni telefoniche a mezzo di fili stesi sulla neve (pp. 323-324). - In questa breve Nota il Prof. Ricco informa della perfetta riuscita degli esperimenti consigliatili dal Janssen per la comunicazione telefonica tra l'osservatorio dell' Etna e quello di Nicolosi, mediante fili stesi semplicemente sulla neve. Il Janssen aggiunge che questo felice esito conferma quanto dalle sue esperienze sul Monte Bianco era stato concluso circa il perfetto potere isolante della neve e del ghiaccio.

BEaroIst L. Leggi della trasparenza della materia per $i$ raggi $X$ (pp. 324.327). - Esperimentando su un gran numero di corpi, in condizioni le più varie circa gli spessori attraversati e $i$ raggi $X$ impiegati, l'A. ba dedotte le leggi prinoipali relative alla trasparenza della materia per i raggi X. Queste leggi sono:

$1^{0} L^{\prime}$ opacità specifica di un corpo sembra indipendente dal suo stato fisico.

$2^{\prime \prime} L^{\prime}$ opacità specifica sembra indipendente dal modo di aggruppamento atomico.

$3^{n} L^{\prime}$ opacità specifica sembra indipendente dallo stato di libertà o di combinazione degli atomi.

L'A. quindi, prendendo per asoisse i pesi atomici e per ordinate gli equivalenti di trasparenza, ha costruito una curva rap- 